\title{
Conhecimento da enfermagem e ações realizadas acerca da prevenção da lesão por pressão: uma revisão integrativa
}

\author{
Nursing knowledge and actions taken \\ about pressure injury prevention: an \\ integrative review
}

\author{
Maristela Silva Melo Santos ${ }^{1}$ (1) \\ Manuela Burke Galrão Alves ${ }^{2}$ (1) \\ Isis Celeste Agra Sousa ${ }^{3}$ (1) \\ Maria Thaís Calasans 4 (1)
}

\begin{abstract}
${ }^{1}$ Autora para correspondência. Escola Bahiana de Medicina e Saúde Pública (Salvador). Bahia, Brasil. maristelasantos.pos@bahiana.edu.br ${ }^{2-4}$ Escola Bahiana de Medicina e Saúde Pública (Salvador). Bahia, Brasil. manuelaalves.pos@bahiana.edu.br, isissousa.pos@bahiana.edu.br,
\end{abstract} mtacalasans@bahiana.edu.br

RESUMO | OBJETIVO: Analisar o conhecimento e ações pelos enfermeiros sobre a prevenção da lesão por pressão. MÉTODO: Revisão integrativa realizada entre os meses de maio e junho de 2020, por meio da busca de artigos nas bases de dados SCIELO, MEDLINE e BDENF, publicados entre 2015 a 2020, com os descritores: "lesão por pressão", "enfermeiro" e "prevenção", utilizando o booleano "and". Foram incluídos artigos originais, em língua portuguesa, publicados na íntegra e disponíveis eletronicamente. RESULTADOS: Foram encontrados 18 artigos, destes apenas 06 responderam à pergunta norteadora. A mudança de decúbito é uma das ações mais realizadas pela equipe de enfermagem como a medida preventiva para o risco de lesão. Isso demonstra uma deficiência no conhecimento dos enfermeiros para a prevenção de lesão por pressão, além da pouca disponibilidade de artigos tratando sobre o assunto. CONSIDERAÇõES FINAIS: Os estudos mostram deficiência no conhecimento do enfermeiro acerca da prevenção de lesão por pressão, destacando a importância de ações preventivas baseadas em protocolos institucionais fundamentados cientificamente.

DESCRITORES: Lesão por pressão. Enfermeiro. Prevenção.

\begin{abstract}
OBJECTIVE: Analyze nurses' knowledge and actions on pressure injury prevention. METHOD: An integrative review carried out between May and June 2020, by searching for articles in the databases SCIELO, MEDLINE, and BDENF, published between 2015 and 2020, with the descriptors: "pressure injury," "nurse," and "prevention," using the Boolean "and." Original articles were included in Portuguese, published in full, and available electronically. RESULTS: 18 articles were found, of which only 06 answered the guiding question. It is noticed a deficiency in the nurses' knowledge for the prevention of pressure injuries and the little availability of articles dealing with the subject. FINAL CONSIDERATIONS: Studies show a deficiency in nurses' knowledge about the prevention of pressure injuries, highlighting the importance of preventive actions based on scientifically based institutional protocols.
\end{abstract}

DESCRIPTORS: Pressure injury. Nurse. Prevention. 


\section{Introdução}

O ambiente hospitalar é um local complexo onde os pacientes estão expostos a diversos fatores de risco, dos quais se pode destacar a mobilidade física comprometida, o estado geral de saúde prejudicado, entre outros ${ }^{1}$.

O desenvolvimento da lesão por pressão (LPP) causa danos significativos aos pacientes, pois dificulta o processo de recuperação funcional, pode causar dor e levar ao desenvolvimento de complicações e ao agravamento de seu estado de saúde. Esse tipo de lesão está associado a internações prolongadas, sepse, mortalidade, elevado custo para a instituição e, ainda, ao aumento da carga de trabalho por parte da equipe de saúde².

A ocorrência de lesão por pressão, nas instituições de saúde no Brasil, precisa ser notificada ao Sistema Nacional de Vigilância Sanitária (SNVS) com o objetivo de mapear cada caso, identificar o problema e planejar ações para a melhoria da assistência prestada aos seus clientes. Os enfermeiros são os profissionais responsáveis não só pela avaliação dos pacientes quanto ao risco desse agravo, mas também pela assistência e prescrição de enfermagem quanto às medidas de prevenção e tratamento, educação de outros profissionais e notificação da ocorrência junto ao SNVS, por meio do sistema informatizado, o Notivisa ${ }^{3}$.

Órgãos internacionais, historicamente, têm contribuído para a construção, consolidação e revisão de diretrizes com recomendações baseadas nas melhores evidências e que auxiliam os profissionais na tomada de decisão e na implementação de condutas para prevenção e tratamento de lesão por pressão, além de auxiliar na elaboração de protocolos institucionais. Dentre eles, destacam-se as ações da National Pressure Injury Advisory Panel (NPIAP)4.

A NPIAP definiu lesão por pressão como danos localizados na pele e/ou tecidos moles subjacentes, geralmente sobre uma proeminência óssea ou relacionada ao uso de dispositivo médico ou a outro artefato. A lesão pode se apresentar em pele íntegra ou como úlcera aberta e ocorre como resultado da pressão intensa e/ou prolongada em combinação com o cisalhamento ${ }^{5}$.
As lesões por pressão são categorizadas para indicar a extensão do dano tissular, sendo classificadas em: estágio 1, quando a pele íntegra apresenta eritema não branqueável; estágio 2 , quando existe perda de pele parcial com exposição de derme; estágio 3, no qual tem-se a perda total da derme com exposição de tecido adiposo e; estágio 4, quando existe a perda da pele e perda tissular com exposição de fáscia muscular, tendão, ligamento, cartilagem ou osso. Normalmente essas lesões acometem mais as áreas de proeminências ósseas, como a região sacra, trocanter e escápula ${ }^{5}$.

Ainda como classificação de lesão, temos: lesão por pressão não classificável é aquela em que ocorre perda de pele em sua espessura total e a perda tissular não pode ser visível, pois encontra-se encoberta por esfacelo ou escara; lesão por pressão relacionada a dispositivo médico, resulta do uso de dispositivos criados e aplicados para fins diagnósticos e terapêuticos, e; lesão por pressão em membrana mucosa é encontrada quando há histórico de uso de dispositivo médico no local do dano ${ }^{5}$.

A ocorrência de lesão por pressão em pacientes internados tem maior incidência em pacientes do gênero masculino (48,5\%), acima de 60 anos $(61,1 \%)$, a região sacral é a mais acometida $(46,4 \%)$, seguida da região calcâneo $(26,76 \%)^{6}$. O aparecimento dessas lesões é multifatorial, estando relacionado com fatores intrínsecos (mobilidade, nutrição e comorbidades) e extrínsecos (uso de fraldas, umidade, aumento de fricção e cisalhamento) ${ }^{3}$.

A avaliação do risco para o desenvolvimento dessas lesões é de fundamental importância para a realização do planejamento e a implementação de medidas para a prevenção e tratamento da lesão por pressão. O registro e a característica da lesão são indispensáveis para a monitorização adequada dos cuidados prestados, a fim de se estabelecer acertadamente medidas de tratamento e melhoria nos cuidados².

O enfermeiro é agente ativo na observação, notificação e tratamento de lesão por pressão. Desse modo, o planejamento do cuidado de enfermagem ao paciente é de sua competência legal, além do conhecimento sobre o assunto, a utilização e aplicação de escalas que permitam avaliar suas evidências. 
O conhecimento científico dos enfermeiros sobre a temática torna-se fundamental, já que cabe a esse profissional identificar os riscos e traçar medidas preventivas para manter a integridade cutânea do seu paciente. Dentre os cuidados prestados, destacam-se a avaliação da pele e preservação da higiene corporal, a realização de mudança de decúbito e a proteção de proeminências ósseas 3 .

Embora existam circunstâncias clínicas nas quais a lesão por pressão é inevitável, a manutenção da integridade da pele do paciente restrito ao leito, ou a recuperação da pele e/ou do tecido subjacente lesado, baseia-se no conhecimento e na aplicação de medidas de cuidado de acordo com as recomendações fundamentadas nas melhores evidências. A elaboração de políticas públicas, assim como a tomada de decisão e o estabelecimento de metas são norteadas por indicadores de qualidade, nos quais está inserida a presença ou a ausência de lesões nas instituições ${ }^{4}$.

É de competência legal do enfermeiro o planejamento do cuidado prestado e direito do paciente a manutenção da integridade cutânea. Visto isso, a temática da prevenção de lesão por pressão é de extrema relevância para os profissionais de enfermagem, principalmente para o enfermeiro, o líder da equipe e responsável pela tomada de decisão ${ }^{7}$.

Dessa forma, este estudo tem como objetivo analisar o conhecimento e as ações utilizadas pelos enfermeiros sobre a prevenção da lesão por pressão.

\section{Método}

Trata-se de um estudo de revisão integrativa, que consiste na construção de uma análise da literatura existente sobre o tema, contribuindo para discussões sobre métodos e resultados de pesquisas, assim como reflexões sobre a realização de futuros estudos. O propósito inicial da revisão integrativa é obter um profundo entendimento de um determinado fenômeno, baseando-se em estudos anteriores ${ }^{8}$.
Para a construção do estudo, foram percorridas as seguintes etapas: escolha do tema; levantamento bibliográfico preliminar; formulação do problema; busca das fontes; leitura do material.

Após a escolha do tema, foi realizado um levantamento prévio da literatura que possibilitou a identificação do problema a ser estudado e elaboração da questão norteadora, que consiste em: Qual o conhecimento e quais ações utilizadas pelos enfermeiros sobre a prevenção da lesão por pressão?

Visando esclarecer esse questionamento, foi realizado um levantamento bibliográfico nas bases de dados: SCIELO, MEDLINE e BDENF, no período de maio a junho de 2020, utilizando os seguintes descritores em Ciências da Saúde (Decs): lesão por pressão, enfermeiro e prevenção, com o operador booleano "and".

Aplicou-se como critérios de inclusão as publicações realizadas entre os anos de 2015 a 2020, em língua portuguesa, e que fossem artigos originais e disponíveis na íntegra eletronicamente. Os critérios de exclusão foram: relatos de experiência, cartas, editoriais, livros, produção duplicada nas bases de dados pesquisadas e trabalhos não relacionados com o escopo do estudo ou que não responderam à questão norteadora desta revisão.

A leitura preliminar, no levantamento bibliográfico, evidenciou a pouca produção cientifica sobres o objeto de estudo, sendo necessário estabelecer o recorte temporal em 05 anos de publicação, tendo em vista melhor análise do objeto de estudo.

Realizado o levantamento de publicações científicas através dos descritores supracitados, foi encontrado um total de 18 artigos, selecionando apenas os que atendiam aos critérios de inclusão. Após coleta de dados, foi realizada a leitura de cada artigo para confirmação de contemplação da pergunta norteadora desta investigação.

Os resultados de cada estudo foram sintetizados e cada artigo recebeu um código de sequência numérica para facilitar a identificação (Estudo 1- E1, Estudo 2- E2, ...). 
Figura 1. Fluxograma da seleção de artigos incluídos na revisão integrativa

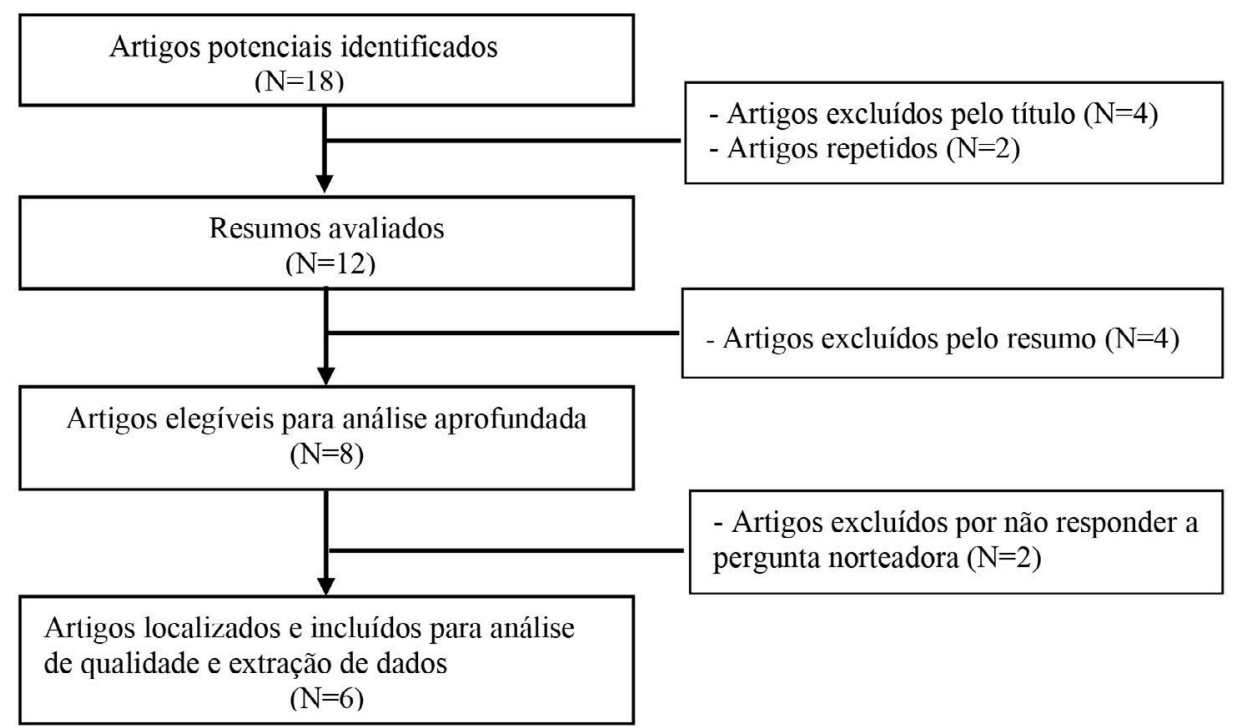

\section{Resultados e discussão}

Os 06 artigos analisados e inseridos no presente estudo foram desenvolvidos no Brasil, nos seguintes períodos: em 2020 (02 artigos), 2019 (01 artigos), 2018 (01 artigo) e em 2017 (02 artigos). A sumarização dos artigos que constituem a amostra da revisão integrativa é apresentada nos quadros 1 e 2.

Quadro 1. Caracterização dos artigos de acordo com a identificação, título, autores, tipo de estudo e objetivo

\begin{tabular}{|c|c|c|}
\hline $\begin{array}{c}\text { Identifi- } \\
\text { cação }\end{array}$ & Títulos/Autores/Ano/Tipo de estudo & Objetivo \\
\hline E1 & $\begin{array}{l}\text { Avaliação e tratamento de lesões por pressão na } \\
\text { estratégia saúde da família. } \\
\text { Souza et al. (2020) } \\
\text { Qualitativo, descritivo, exploratório. }\end{array}$ & $\begin{array}{l}\text { Conhecer como os enfermeiros atuam na } \\
\text { avaliação e tratamento de LPP no contexto } \\
\text { da ESF. }\end{array}$ \\
\hline E2 & $\begin{array}{l}\text { Cultura organizacional: prevenção, tratamento e } \\
\text { gerenciamento de risco da lesão por pressão. } \\
\text { Souza, Loureiro e Batiston (2020) } \\
\text { Transversal. }\end{array}$ & $\begin{array}{l}\text { Identificar os fatores facilitadores e } \\
\text { dificultadores para a prevenção e } \\
\text { tratamento da LPP na gestão da assistência } \\
\text { ao paciente hospitalizado. }\end{array}$ \\
\hline E3 & $\begin{array}{l}\text { Conhecimento de enfermeiros sobre prevenção e } \\
\text { cuidados de lesão por pressão. } \\
\text { Sousa e Faustino (2019) } \\
\text { Transversal descritivo. }\end{array}$ & $\begin{array}{l}\text { Identificar o conhecimento dos enfermeiros } \\
\text { assistenciais quanto à prevenção e aos } \\
\text { cuidados com LPP em unidades de CM e CC } \\
\text { de um hospital universitário em Brasília. }\end{array}$ \\
\hline E4 & $\begin{array}{l}\text { Prevenção de lesão por pressão: Ações prescritas por } \\
\text { enfermeiros de centros de terapia intensiva. } \\
\text { Mendonça, Loureiro, Frota e Souza (2018) } \\
\text { Transversal descritivo e analítico. }\end{array}$ & $\begin{array}{l}\text { Descrever as ações de enfermagem } \\
\text { prescritas por enfermeiros para a } \\
\text { prevenção de LPP e sua ocorrência em CTI. }\end{array}$ \\
\hline E5 & $\begin{array}{l}\text { Conhecimento da equipe de enfermagem sobre } \\
\text { prevenção de úlceras por pressão. } \\
\text { Galvão, Serique, Santos e Nogueira (2017) }{ }^{13} \text {. } \\
\text { Descritivo e exploratório. }\end{array}$ & $\begin{array}{l}\text { Descrever e analisar os conhecimentos da } \\
\text { equipe de enfermagem acerca da } \\
\text { classificação, avaliação e medidas de } \\
\text { prevenção de UPP em pacientes de um } \\
\text { Hospital Universitário da cidade de Manaus. }\end{array}$ \\
\hline E6 & $\begin{array}{l}\text { Ações de enfermagem antes e após um protocolo de } \\
\text { prevenção de lesões por pressão em terapia intensiva } \\
\text { Vasconcelos e Caliri (2017) } \\
\text { Observacional, prospectivo, comparativo com } \\
\text { abordagem quantitativa. }\end{array}$ & $\begin{array}{l}\text { Analisar as ações dos profissionais de } \\
\text { enfermagem, antes e após utilização de } \\
\text { protocolo de prevenção de LPP em UTI. }\end{array}$ \\
\hline
\end{tabular}

Fonte: Dados da pesquisa 
Quadro 2. Caracterização dos artigos de acordo com a identificação, resultados e conclusão

\begin{tabular}{|c|c|c|}
\hline $\begin{array}{c}\text { Identifi- } \\
\text { Cação }\end{array}$ & Principais resultados & Conclusão \\
\hline E1 & $\begin{array}{l}\text { Elencaram-se as seguintes categorias: Participação em } \\
\text { formação específica sobre lesão por pressão; aplicação de } \\
\text { métodos de avaliação de lesões por pressão; indicação, } \\
\text { utilização e disponibilidade de coberturas para a prevenção } \\
\text { e tratamento de lesões por pressão e orientações a } \\
\text { pacientes e familiares sobre os cuidados com lesões por } \\
\text { pressão. }\end{array}$ & $\begin{array}{l}\text { O enfermeiro necessita possuir o } \\
\text { conhecimento teórico-prático para } \\
\text { que, juntamente a equipe } \\
\text { multiprofissional e à família, promova } \\
\text { o cuidado na prevenção e tratamento } \\
\text { necessário a estes pacientes. }\end{array}$ \\
\hline E2 & $\begin{array}{l}\text { Entre os fatores identificados, destaca-se que } 59 \% \text { dos } \\
\text { entrevistados desconhecem o protocolo de prevenção de } \\
\text { LP, } 27 \% \text { não utilizam a avaliação clínica para } \\
\text { dimensionamento diário dos profissionais, mais de } 52 \% \\
\text { acreditam não existir elementos facilitadores e } 76 \% \\
\text { afirmam existir elementos dificultadores para a prevenção } \\
\text { de LP. Quanto ao tratamento, pouco mais de } 60 \% \text { referem } \\
\text { que o paciente e a lesão são avaliados por enfermeiros, } \\
\text { sendo que } 54 \% \text { dos procedimentos são prescritos pelo } \\
\text { médico e } 46 \% \text { da terapêutica é executada por técnicos de } \\
\text { enfermagem. }\end{array}$ & $\begin{array}{l}\text { A prevenção e o tratamento da LP } \\
\text { necessitam da gestão compartilhada, } \\
\text { com ações integradas entre os } \\
\text { executores da assistência. }\end{array}$ \\
\hline E3 & $\begin{array}{l}\text { A amostra foi de } 38 \text { enfermeiros, na qual } 78,9 \% \text { acertaram } \\
\text { entre } 70 \text { a } 89 \% \text { do instrumento e somente dois enfermeiros } \\
(5,2 \%) \text { obtiveram nota igual ou maior a } 90 \% \text { de acerto. Os } \\
\text { itens de menor acerto estão relacionados ao uso de } \\
\text { dispositivos, como luva d'agua ( } 24,6 \%) \text {, almofadas }(23,6 \%) \text { e } \\
\text { em relação a posicionamento e reposicionamento, além da } \\
\text { massagem em proeminências ósseas. }\end{array}$ & $\begin{array}{l}\text { Há um déficit do conhecimento da } \\
\text { equipe de enfermagem deste hospital, } \\
\text { o que pode comprometer diretamente } \\
\text { na assistência, principalmente do } \\
\text { paciente em risco para LPP. }\end{array}$ \\
\hline E4 & $\begin{array}{l}\text { Foi encontrada associação estatística entre as ações de } \\
\text { mudança de decúbito, aplicação de cobertura hidrocolóide } \\
\text { em região sacral, realização de higiene externa, troca de } \\
\text { fixação do cateter orotraqueal e/ou cateter nasoenteral e } \\
\text { inspeção de pele com ausência de lesões por pressão. A } \\
\text { ocorrência de lesões por pressão foi encontrada em 49\% } \\
\text { dos clientes em ambas as instituições. }\end{array}$ & $\begin{array}{l}\text { A elaboração e implementação de } \\
\text { protocolos, e o acompanhamento dos } \\
\text { registros e dos grupos de maior risco } \\
\text { são estratégias que direcionam a } \\
\text { prescrições de ações preventivas } \\
\text { adequadas para lesões por pressão. }\end{array}$ \\
\hline E5 & $\begin{array}{l}\text { As médias globais de acertos foram } 63,4 \% \text { para os } \\
\text { técnicos/auxiliares de enfermagem e } 51,4 \% \text { para } \\
\text { enfermeiros, com diferença estatisticamente significativa } \\
\text { entre os grupos somente para a categoria de prevenção de } \\
\text { UP ( } p<0,001) \text {. }\end{array}$ & $\begin{array}{l}\text { Déficit de conhecimentos sobre a } \\
\text { prevenção de UP entre enfermeiros e } \\
\text { técnicos/auxiliares de enfermagem, } \\
\text { tornando mandatória a capacitação } \\
\text { desses profissionais. }\end{array}$ \\
\hline E6 & $\begin{array}{l}\text { Após uso do protocolo, observou-se maior frequência das } \\
\text { ações: avaliação do risco para lesões por pressão nos dias } \\
\text { subsequentes à admissão. }\end{array}$ & $\begin{array}{l}\text { A maior frequência de ações } \\
\text { preventivas após o uso do protocolo } \\
\text { demonstra a importância dessa } \\
\text { ferramenta na adoção das } \\
\text { recomendações baseadas em } \\
\text { evidências cientificas pelos } \\
\text { profissionais. }\end{array}$ \\
\hline
\end{tabular}

Fonte: Dados da pesquisa 
Os estudos analisados são de diversas regiões do Brasil, sendo E1- Sul (Rio Grande do Sul), E2- Centro Oeste (Mato Grosso do Sul), E3- Distrito Federal (Brasília), E4- Sudeste (Rio de Janeiro), E5- Norte (Manaus) e E6- Nordeste (João Pessoa). Desta forma, foram encontradas ações de prevenção de lesão por pressão com representatividade de todas as regiões do território brasileiro. Ademais, os estudos identificados envolvem enfermeiros de todos os níveis da assistência.

Todos os artigos analisados abordaram a temática "prevenção de lesão por pressão pelos enfermeiros", mostrando ações preventivas, utilização de protocolos e o nível de conhecimento desses profissionais.

O Enfermeiro é membro da equipe multiprofissional de saúde, líder da equipe de enfermagem e responsável pela tomada de decisão na prática do cuidado ao paciente hospitalizado, visando à busca da qualidade da assistência. Para garantir a qualidade assistencial, faz-se necessário conhecimento científico, entretanto, todos os estudos analisados mostraram que o conhecimento do Enfermeiro acerca da prevenção de LPP ainda é deficiente ${ }^{11}$.

Segundo Souza et al. 9 , as LPP são consideradas eventos adversos e, por serem evitáveis, são inseridas no contexto da qualidade e segurança assistencial ao paciente. Dessa forma, revelam uma deficiência da qualidade na assistência prestada, quando ocorrem com frequência.

Os artigos estudados também discutem que a elaboração e a implementação de protocolos, o acompanhamento dos registros e uma gestão compartilhada com ações integradas entre os executores da assistência são estratégias que direcionam a prescrição que previnem lesões por pressão. Todos os artigos discorrem quanto à relevância de protocolos para nortear as ações de enfermagem, principalmente para a prevenção da lesão por pressão.

Para que haja a redução na ocorrência de lesão por pressão é necessária a elaboração de estratégias de fortalecimento das práticas assistenciais, e é de suma importância a utilização de diretrizes e implantação de protocolos de prevenção para nortear os profissionais de saúde ${ }^{10}$.
Mendonça et al. ${ }^{12}$, ao descreverem as ações de enfermagem para a prevenção de lesões por pressão em centros de terapia intensiva, mostraram que foram encontradas associações estatísticas entre as ações preventivas implementadas baseadas em protocolos e evidências com a ausência de lesões por pressão.

Dentre as ações de enfermagem mais encontradas, têm-se a mudança de decúbito (a cada duas horas) e manutenção da pele limpa e seca, como principais medidas de prevenção de LPP. Porém, notou-se uma aleatoriedade na elaboração das prescrições e uma ocorrência de lesão por pressão em $49 \%$ dos clientes em ambas as instituições avaliadas. A não uniformização das ações para prevenção de LPP está relacionada à falta de conhecimento adequado dos enfermeiros acerca das medidas preventivas ${ }^{10}$.

Souza, Loureiro e Batiston ${ }^{10}$ defendem uma gestão compartilhada entre os executores das ações de prevenção de LPP e entra em concordância com Mendonça et al. $\frac{12}{2}$ quando fala da importância do uso de diretrizes e implantação de protocolos de prevenção como estratégias de melhoria para a qualidade da assistência.

Os estudos ainda trazem resultados que revelam que as instituições não oferecem atividades educativas sobre o tema em questão aos seus profissionais, com grande dificuldade de investir na contínua qualificação do sujeito. A educação continuada tem como objetivo proporcionar ao profissional de saúde conhecimentos específicos e atualizações constantes, incluindo a prevenção de agravos, buscando a excelência na qualidade da assistência prestada ao paciente ${ }^{13}$.

Diante disso, Vasconcelos e Caliri14 avaliam as ações dos profissionais de enfermagem, antes e após a utilização de protocolos de prevenção de LPP, e concluem a importância dessa ferramenta. A qual traz resultados significativos, considerando as ações realizadas pelos profissionais durante o banho no leito, recomendadas para o controle dos fatores de risco, antes e após divulgação de protocolo. Os dados revelaram mudança no comportamento e aumento no número de ações preventivas em todas as variáveis observadas após a utilização do protocolo. 
Os estudos de Souza, Loureiro e Batiston ${ }^{10}$, Sousa e Faustino ${ }^{11}$ e Mendonça et al..$^{12}$ mostram que a aplicação de escalas de avaliação de risco para o desenvolvimento de LPP, combinada com o raciocínio clínico, pode auxiliar os profissionais a estabelecerem as intervenções mais adequadas para o paciente. As escalas de Braden, Waterlow e Norton são instrumentos validados e recomendados como sugestão para avaliar os riscos do paciente desenvolver LPP ${ }^{14}$. Porém, deixam explícito que a utilização dessas escalas é realizada de forma aleatória, sem conhecimento específico, fazendo com que seu resultado não corresponda à verdadeira necessidade do paciente.

Ao avaliar o nível de informação dos enfermeiros acerca da prevenção de LPP, dois estudos trouxeram deficiência de conhecimentos específicos. Sousa e Faustino ${ }^{11}$ e Galvão et al. ${ }^{13}$ utilizaram como instrumento de avaliação a aplicação de questionários previamente validados, com perguntas para serem respondidas como verdadeiro ou falso, tratando de avaliação, classificação da LPP e prevenção. Em ambos os estudos, os resultados foram semelhantes, chamando atenção para o despreparo do profissional da enfermagem em cuidar dos pacientes portadores de feridas, déficit de conhecimento sobre a prevenção, a avaliação e a classificação da LPP, e a necessidade de atualização, visto que o conhecimento está diretamente ligado à qualidade da assistência prestada.

Medidas de prevenção condenadas pela NPIAP, como a utilização de luvas d'água ou de ar para aliviar a pressão nos calcâneos e almofadas, tipo rodas d'água para auxiliar na prevenção da LPP, foram apontadas pelos enfermeiros como estratégia de cuidado, o que reforça a falta de conhecimento ou falta de atualização destes profissionais sobre a temática. Esses fatos corroboram para uma deficiência nas ações da equipe de enfermagem, expondo o paciente a riscos e que propícia à ocorrência de eventos adversos ${ }^{15}$.

As estratégias de prevenção traçadas por Souza et al. 9 , que mais se aproximam do que traz a Agência Nacional de Vigilância Sanitária (Anvisa) quanto a Prática Segura para prevenção de LPP, são: Realização de avaliação de risco de todos os pacientes antes e durante a internação; Realização de avaliação criteriosa da pele pelo menos uma vez por dia, principalmente em proeminências ósseas; Uso de colchão especial e almofadas para a distribuição da pressão;
Uso de apoio na altura da panturrilha; Manutenção da higiene corporal, mantendo pele limpa e seca; Hidratação diária da pele com hidratante e umectantes, quando necessário; Uso de barreiras protetoras da umidade excessiva, quando necessário, como por exemplo: creme barreira, película semipermeável e espuma de poliuretano; Mudança de posição a cada duas horas para reduzir a pressão local; Manutenção da cabeceira a $30^{\circ}$ e; Orientação da família e paciente na prevenção e tratamento da LPP 13 .

No que se refere às ações preventivas, não basta apenas ter o conhecimento, é preciso aprimorar habilidades e atitudes. É primordial que a equipe de enfermagem execute as medidas com responsabilidade, a fim de diminuir o risco de um indivíduo desenvolver LPP ${ }^{16}$.

O enfermeiro tem como papel fundamental na prevenção de LPP a identificação dos fatores de risco, implementação de medidas preventivas e realização de assistência com qualidade. Para isso, ele deve possuir conhecimentos científicos em relação aos estágios da LPP, uma vez que ele prescreve as ações/ medidas que serão adotadas como cuidados preventivos para a equipe de enfermagem ${ }^{17}$. As recomendações preconizadas pelas novas diretrizes da NPIAP e da ANVISA irão conduzir o enfermeiro na tomada de decisão sobre a intervenção adequada ${ }^{18}$.

Souza, Loureiro e Batiston ${ }^{10}$ trazem dados relacionados à avaliação e tratamento da LPP na estratégia saúde da família e confirma que o enfermeiro precisa possuir o conhecimento teórico e prático, promovendo o cuidado na prevenção e tratamento necessário aos pacientes, juntamente com a equipe multiprofissional e à família.

O enfermeiro é fundamental nesse processo e o mais apropriado para gerir essa ação e elevar a qualidade da assistência de saúde. A prevenção da lesão por pressão está diretamente ligada à ação da equipe de enfermagem, o que torna essencial o conhecimento científico para a promoção de cuidados visando à prevenção dessas lesões. Estudos trazem como dificultadores para a utilização das medidas preventivas: a falta de conhecimento do profissional, deficiência da educação continuada nas instituições e a ausência da implantação da Sistematização da Assistência de Enfermagem baseadas nas diretrizes de boas práticas ${ }^{15}$. 


\section{Considerações finais}

De uma forma geral, os estudos analisados mostram deficiência no conhecimento do enfermeiro acerca da prevenção de LPP, destacando a importância de ações preventivas baseadas em protocolos institucionais fundamentados cientificamente. Os enfermeiros, ao se apropriarem do conhecimento da etiologia das lesões por pressão, dos fatores de risco associados ao seu desenvolvimento e da utilização de protocolos específicos validados facilmente, promovem a integridade cutânea, fazendo julgamentos clínicos e aplicações de medidas preventivas.

É de fundamental importância que a enfermagem tenha o conhecimento sobre todo o processo que envolve o tratamento do paciente, o desenvolvimento de protocolos que sigam uma ordem na avaliação, classificação, acompanhamento e reavaliação é a chave para o sucesso nas estratégias de prevenção.

O presente estudo apresentou limitações quanto à sua amostra, tendo em vista os poucos estudos, publicados nos últimos cinco anos, que abordassem a temática de conhecimento e ações dos enfermeiros voltadas para a prevenção de lesão por pressão. Não foram levados em consideração os estudos que abordassem o tratamento de lesões, para que pudéssemos manter o foco no conhecimento do enfermeiro e nas medidas adotadas apenas para o não aparecimento das lesões.

Espera-se que os achados possam ser aplicados na assistência aos pacientes, subsidiando o enfermeiro na implantação de medidas preventivas para evitar a ocorrência de lesão por pressão.

\section{Contribuições dos autores}

Santos MSM, Alves MBG e Sousa ICA participaram da concepção, delineamento, coleta, análise e interpretação dos dados e redação do artigo. Calasans MT participou da concepção, orientação, delineamento, coleta, interpretação dos dados, redação e revisão final do artigo cientifico.

\section{Conflitos de interesses}

Nenhum conflito financeiro, legal ou político envolvendo terceiros (governo, empresas e fundações privadas, etc.) foi declarado para nenhum aspecto do trabalho submetido (incluindo, mas não se limitando a subvenções e financiamentos, participação em conselho consultivo, desenho de estudo, preparação de manuscrito, análise estatística, etc.).

\section{Referências}

1. Vocci MC. Lesão por pressão na população pediátrica: estudo de coorte com aplicação da escala Braden Q. [dissertação] [Internet]. Botucatu: Universidade Estadual Paulista "Júlio de Mesquita Filho"; 2017. [citado em 2020 abr. 09]. http://hdl.handle. net/11449/150293

2. Olkoski E, Assis GM. Aplicação de medidas de prevenção para úlceras por pressão pela equipe de enfermagem antes e após uma campanha educativa. Esc. Anna Nery [Internet]. 2016;20(2):363-9. Disponível em: https://www.scielo.br/scielo. php?pid=S1414-81452016000200363\&script=sci_abstract\&tIng=pt

3. Agência Nacional de Vigilância Sanitária. NOTA TÉCNICA GVIMS/ GGTES N03/2017: Práticas seguras para prevenção de Lesão por Pressão em serviços de saúde [Internet]. 2018. [acesso em 2020 jun. 02]. Disponível em: https://www.gov.br/anvisa/pt-br/ centraisdeconteudo/publicacoes/servicosdesaude/notas-tecnicas/ nota-tecnica-gvims-ggtes-no-03-2017.pdf/view

4. Moraes JT, Borges EL, Lisboa CR, Cordeiro DCO, Rosa EG, Rocha NA. Conceito e classificação de lesão por pressão: atualização do National Pressure Ulcer Advisory Panel. Enferm. Cent. O. Min. 2016;6(2):2292-306. https://doi.org/10.19175/recom.v6i2.1423

5. Caliri MHL, Santos VLCG, Mandelbaum MHS, Costa IG. Classificação das lesões por pressão. Consenso NPUAP. Adaptada Culturalmente para o Brasil. Associação Brasileira de Estomaterapia, Associação Brasileira de Enfermagem em Dermatologia. [citado em 2017 mai 17]. Disponível em: https:// www.ibes.med.br/classificacao-das-lesoes-por-pressao-consensonpuap-2016-adaptada-culturalmente-ao-brasil//

6. Teixeira AKS, Nascimento TS, Sousa ITL, Sampaio LRL, Pinheiro ARM. Incidência de lesões por pressão em Unidade de Terapia Intensiva em hospital com acreditação. ESTIMA [Internet]. 2017;15(3). Disponível em: https://www.revistaestima.com.br/ estima/article/view/545 
7. Marques LG, Vieira MLC, Pereira SRM. Artigo Original 1 - A Construção do Conhecimento dos Enfermeiros Perante a Nova Classificação da Úlcera por Pressão. ESTIMA [Internet]. 2016;11(1). Disponível em: https://www.revistaestima.com.br/estima/article/ view/80

8. Turato ER. Introdução à metodologia da pesquisa clínicoqualitativa - definições e principais características. Rev. Portuguesa de Psicossomática [Internet]. 2000;2(1):93-108. Disponível em: https://www.redalyc.org/articulo.oa?id=28720111

9. Souza E, Rodrigues NH, Silva LGA, Silva DM, Oliveira SG, Souza LM. Avaliação e tratamento de lesões por pressão na Estratégia Saúde da Família. Rev. Enferm. UFPE on-line. 2020;14:e243522. https://doi.org/10.5205/1981-8963.2020.243522

10. Souza MC, Loureiro MDR, Batiston AP. Cultura organizacional: prevenção, tratamento e gerenciamento de risco da lesão por pressãoy. Rev. Bras. Enferm. 2020;73(3):e20180510. http://dx.doi. org/10.1590/0034-7167-2018-0510

11. Sousa RC, Faustino AM. Conhecimento de enfermeiros sobre prevenção e cuidados de lesão por pressão. Rev. Fun. Care Online [Internet]. 2019;11(4):992-7. Disponível em: https://pesquisa. bvsalud.org/portal/resource/pt/biblio-1005821

12. Mendonça PK, Loureiro MDR, Frota OP, Souza AS. Prevenção de lesão por pressão: ações prescritas por enfermeiros de centros de terapia intensiva. Texto contexto - enferm. 2018;27(4):e4610017. http://doi.org/10.1590/0104$\underline{07072018004610017}$
13. Galvão NS, Serique MAB, Santos VLCG, Nogueira PC. Conhecimentos da equipe de enfermagem sobre prevenção de úlceras por pressão. Rev. Bras. Enferm. 2017;70(2):294-300. http:// dx.doi.org/10.1590/0034-7167-2016-0063

14. Vasconcelos JMB, Caliri MHL. Ações de enfermagem antes e após um protocolo de prevenção de lesões por pressão em terapia intensiva. Esc. Anna Nery [Internet]. 2017;21(1):e20170001. Disponível em: https://www.scielo.br/scielo.php?pid=S1414$81452017000100201 \&$ script $=$ sci abstract\&tlng=pt

15. Santos GMG, Rocha RRS, Melo AFS, Passos TS. O enfermeiro frente à prevenção de lesão por pressão: Revisão intergrativa. Journal of Health Connection [Internet]. 2018;3(2):60-71. Disponível em: http://revistaadmmade.estacio.br/index.php/ journalhc/article/download/4520/47964961

16. Campoi ALM, Engel RH, Stacciarini TSG, Cordeiro ALPC, Melo AF, Rezende MP. Educação permanente para boas práticas na prevenção de lesão por pressão: quase-experimento. Rev. Bras. Enferm. 2019;72(6):1646-52. https://doi.org/10.1590/0034-7167$\underline{2018-0778}$

17. Frazão JM, Moraes FTR, Reis MNS, Silva SL. A Abordagem do enfermeiro na prevenção de feridas em pacientes hospitalizados. REAID [Internet]. 2019;88(26):1. Disponível em: https:// revistaenfermagematual.com/index.php/revista/article/view/307

18. Correia ASB, Santos IBC. Lesão por pressão: medidas terapêuticas utilizadas por profissionais da enfermagem. RBCS [Internet]. 2019;23(1):33-42. Disponível em: https://periodicos. ufpb.br/index.php/rbcs/article/view/36793-p4 